\title{
An Australian Indigenous community-led suicide intervention skills training program: community consultation findings
}

\author{
Bushra Nasir ${ }^{1 *} \mathbb{D}$, Steve Kisely ${ }^{1,2}$, Leanne Hides ${ }^{3}$, Geetha Ranmuthugala ${ }^{1,4}$, Sharon Brennan-Olsen ${ }^{5}$, \\ Geoffrey C. Nicholson ${ }^{1}$, Neeraj S. Gill', Noel Hayman', Srinivas Kondalsamy-Chennakesavan ${ }^{1}$ and Maree Toombs ${ }^{1}$
}

\begin{abstract}
Background: Little is known of the appropriateness of existing gatekeeper suicide prevention programs for Indigenous communities. Despite the high rates of Indigenous suicide in Australia, especially among Indigenous youth, it is unclear how effective existing suicide prevention programs are in providing appropriate management of Indigenous people at risk of suicide.

Methods: In-depth, semi-structured interviews and focus groups were conducted with Indigenous communities in rural and regional areas of Southern Queensland. Thematic analysis was performed on the gathered information.

Results: Existing programs were time-intensive and included content irrelevant to Indigenous people. There was inconsistency in the content and delivery of gatekeeper training. Programs were also not sustainable for rural and regional Indigenous communities.

Conclusions: Appropriate programs should be practical, relevant, and sustainable across all Indigenous communities, with a focus on the social, emotional, cultural and spiritual underpinnings of community wellbeing. Programs need to be developed in thorough consultation with Indigenous communities. Indigenous-led suicide intervention training programs are needed to mitigate the increasing rates of suicide experienced by Indigenous peoples living in rural and remote locations.
\end{abstract}

Keywords: Indigenous, Community-consultation, Suicide prevention, Suicide intervention

\section{Background}

Indigenous Australians have twice the suicide rate of non-Indigenous Australians; and the rates are four times higher among Indigenous youth [1,2]. Suicide rates also tend to increase with remoteness so that Indigenous populations in very remote areas have twice the suicide rate as those in major cities [3]. This is of concern given Indigenous Australians comprise a large proportion of the population of very remote and remote residents in Australia [3].

Despite the high burden of suicide amongst Indigenous Australians there are relatively few Indigenous-specific suicide prevention programs

\footnotetext{
* Correspondence: b.nasir@uq.edu.au

'Rural Clinical School, Faculty of Medicine, The University of Queensland,

Toowoomba, QLD, Australia

Full list of author information is available at the end of the article
}

available [4]. Indigenous communities share elements of culture, language and heritage, and it is essential that suicide prevention and intervention programs encourage connectedness, facilitate assimilation, and maintain cultural heritage [5]. Reports have shown that a community-led participatory approach is required to develop a comprehensive, effective and long-term program that can tackle the multi-dimensional aspects of suicide prevention and intervention [6].

\section{Gatekeeper training}

Gatekeeper suicide prevention training teaches specific groups of people the knowledge, attitudes and skills necessary to identify people at risk of suicide and implement appropriate preventive interventions [7]. Frontline gatekeeper education and intervention training is one of the most effective suicide prevention strategies available 
[7, 8]. International studies have shown that gatekeeper training programs can contribute to significant improvements in trainer skills in suicide prevention [9-13]. However, other research has questioned the use of Western gatekeeper training programs as an effective suicide prevention tool for Indigenous communities [14, 15]. There is therefore a need for a culturally tailored, diverse, and systematic suicide prevention program targeting Australian Indigenous people at risk of suicide. An effective early intervention program aimed at increasing gatekeepers' ability to identify suicide risk and implement effective suicide prevention strategies could enable Indigenous communities to maintain sustainable suicide prevention programs $[9,10]$.

\section{The Indigenous Network Suicide Intervention Skills Training project}

The Indigenous Network Suicide Intervention Skills Training (INSIST) project aims to design, implement and evaluate a new multi-faceted, culturally-tailored intervention training program for preventing suicide among Indigenous people, especially Indigenous youth. The project has three aims: 1) to increase the knowledge and awareness of suicide risk factors; 2) develop a sense of connectedness among gatekeepers, at-risk groups, health services and support groups; and 3) to develop knowledge and awareness of professional and ethical responsibilities related to suicide risk among Indigenous communities.

The first phase of the project was to develop an intervention training package, drawing on existing models, through community based participatory research. This paper reports the findings from consultations held with community members and key stakeholders for the purpose of a) reviewing existing Indigenous gatekeeper training packages; b) assessing cultural appropriateness of the content in the programs; and c) identifying the key components of a culturally appropriate gatekeeper training program.

\section{Methods}

\section{Research approach}

Community based participatory research (CBPR), in which consultations are held with relevant parties and organisations as well as members of the broader community [16], is highly beneficial for addressing complex issues, particularly in Indigenous communities $[17,18]$. We undertook such consultations in Indigenous communities in rural and regional Southern Queensland, Australia using an approach informed by guidelines from the Consolidated Criteria for Reporting Qualitative Research (COREQ) [19] and further explained by the Standards for Reporting Qualitative Research (SRQR) [20].
Involvement of Indigenous researchers, as well as participation of Indigenous community members and organisations were critical to the study. Indigenous as well as non-Indigenous community members, stakeholders and service providers all played a key role in the consultations. Consultations involved both one-onone interviews, as well as focus groups with larger groups of people. Detailed participant demographics were not recorded to maintain confidentiality. However, consultations were carried out with both nonIndigenous and Indigenous people, and people representative from all demographic backgrounds of the consultation settings. Community concerns were prioritised, and cultural sensitivity and relevance was maintained during the consultation due to the nature of the sensitive topic of discussion. The resulting outcomes of each consultation were interpreted and discussed with participants to ensure the validity of the generated outcomes.

\section{Data analysis}

Qualitative data were generated from unidentified notes taken during in-depth, semi-structured consultations allowing for themes and ideas to be relayed, and the discovery of elaborate information important to participants. Questions explored the current perceptions of gatekeeper training programs for Indigenous people, especially youth; i.e. "Can you comment on the cultural appropriateness of current suicide prevention training programs?" and "Can you tell me about some components that would be beneficial for suicide prevention and intervention as part of a culturally appropriate gatekeeper training program?"

On completion of each consultation, discussion with community members and investigators identified significant ideas and observations that were considered fundamental to the consultation process; upon reaching consensus, outcomes were documented. The community members played a key role in checking the validity and relevance of the resulting outcomes and consultation themes. Indigenous communities and organisations were provided the opportunity to 'own' the date collected and given a chance to 'voice' their concerns. Anonymous, systematic, thematic analysis was then performed with the information gained from community. Quality analysis assessment $[19,20]$ was conducted by the investigators to enhance and substantiate the analysis outcomes.

\section{Results}

Study sample

Initially, the research team spent time building rapport with communities where suicide prevalence was known to be high. This led to consultations with multiple 
community members, health care service providers, volunteer workers, and key organisations providing mental health as well as physical health support programs (Table 1). These frontline service providers were deemed

Table 1 List of key organisations from which representatives were consulted

\begin{tabular}{|c|c|c|}
\hline Name of organisation & Location & ASGS-RA \\
\hline $\begin{array}{l}\text { Charleville \& Western Areas Aboriginal \& } \\
\text { Torres Strait Islanders Corp for Health } \\
\text { (CWAATSICH) }\end{array}$ & Roma & RA 3 \\
\hline QAS - Queensland Ambulance Service & Roma & RA 3 \\
\hline $\begin{array}{l}\text { Queensland Department of Allied } \\
\text { Health Services }\end{array}$ & Roma & RA 3 \\
\hline $\begin{array}{l}\text { Maranoa Regional Council } \\
\text { (Community Support/Youth) }\end{array}$ & Roma & RA 3 \\
\hline Standby Response Services & Roma & RA 3 \\
\hline Gateway To Training & Goondiwindi & RA 3 \\
\hline DRUGARM & Goondiwindi & RA 3 \\
\hline $\begin{array}{l}\text { Centre Care Toowoomba -Partners } \\
\text { in Recovery (PIR) }\end{array}$ & St George & RA 4 \\
\hline St. George Aboriginal Housing Company & St George & RA 4 \\
\hline QPS-Queensland Police Service & St George & RA 4 \\
\hline RFDS - Royal Flying Doctor Service & St George & RA 4 \\
\hline $\begin{array}{l}\text { Charleville \& Western Areas Aboriginal \& } \\
\text { Torres Strait Islanders Corp for Health } \\
\text { (CWAATSICH) }\end{array}$ & Charleville & RA 5 \\
\hline Community Allied Health Services & Charleville & RA 5 \\
\hline QPS - Queensland Police Service & Charleville & RA 5 \\
\hline Charleville Neighbourhood Centre & Charleville & RA 5 \\
\hline QAS -Queensland Ambulance Service & Charleville & RA 5 \\
\hline Far West Family Violence & Charleville & RA 5 \\
\hline Royal Flying Doctor Service & Charleville & RA 5 \\
\hline Lifeline Darling Downs & Charleville & RA 5 \\
\hline PHaMs -Personal Helpers and Mentors & Charleville & RA 5 \\
\hline QPS - Queensland Police Service & Charleville & RA 5 \\
\hline Queensland Health- HOPE Project & Charleville & RA 5 \\
\hline $\begin{array}{l}\text { Centre Care Toowoomba- Domestic } \\
\text { \& Family Violence }\end{array}$ & Cunnamulla & RA 5 \\
\hline $\begin{array}{l}\text { Centacare Toowoomba -Partners } \\
\text { in Recovery (PIR)Far West Family } \\
\text { Violence Service }\end{array}$ & Cunnamulla & RA 5 \\
\hline Paroo Regional Council & Cunnamulla & RA 5 \\
\hline QPS - Queensland Police Service & Cunnamulla & RA 5 \\
\hline Royal Flying Doctor Service & Cunnamulla & RA 5 \\
\hline PHaMs -Personal Helpers and Mentors Service & Cunnamulla & RA 5 \\
\hline $\begin{array}{l}\text { Cunnamulla Aboriginal Corporation for } \\
\text { Health-Primary Health Centre (CACH) }\end{array}$ & Cunnamulla & RA 5 \\
\hline $\begin{array}{l}\text { Queensland Country Practice Rural Remote } \\
\text { Medical support-Cunnamulla Medical Clinic }\end{array}$ & Cunnamulla & RA 5 \\
\hline
\end{tabular}

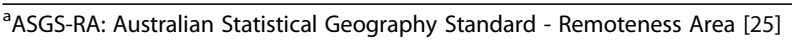

to be most at need for training in the identification of at risk individuals and suicide prevention. A total of 29 consultations were conducted that ranged in duration from $10 \mathrm{~min}$ to $2 \mathrm{~h}$; some consolations were conducted collectively with groups of people and therefore took longer than individual consultations.

\section{Review of existing models of Indigenous gatekeeper training packages}

All participants had previously undertaken generic gatekeeper suicide prevention training or had detailed knowledge in the area. Participants highlighted the problematic nature of the current delivery of gatekeeper training programs by a number of entities funded under different schemes, without any consistency. The majority indicated that existing gatekeeper trainings were "lengthy and contained irrelevant information for Indigenous people". This was considered "burdensome", given the limited resources available in regional areas. They reported many people were "unaware of the services available", which often resulted in suicidal people "not accessing the help available and the local communities trying to manage the risk of suicide themselves". There was a strong desire for community appropriate training, and supporting tools to empower and sustain the training provided. Participants indicated that they needed "something that they can use straight away and don't need to spend too much time learning or that is too hard for anyone."

\section{Identification of culturally inappropriate content in existing gatekeeper programs}

Participants considered existing models were not culturally tailored to local Indigenous communities and indicated that they "don't like what is currently out there as it doesn't really work". Shortfalls included the lack of appropriate language and the use of culturally irrelevant scenarios. "No one can really understand them and know how to use it in real life." Communities indicated there was still a sense of "shame" when talking about suicide related issues. It was acknowledged that current training and knowledge within the community for identifying and preventing suicide was very low, due to gaps in training packages. Furthermore, it was indicated that little consultation with Indigenous communities was carried out and cultural training was often not provided to the trainers.. "They (the trainers) don't really know our culture." Current models for suicide prevention were deemed to be "too lengthy for busy staff and too expensive", which deterred communities from accepting these training packages as appropriate or useful. It was also recognized that existing models were not sustainable within these rural and regional communities, due to the 
lack of staff and resources, and "socially unacceptable content" surrounding suicide for Indigenous communities.

\section{Key components required for a culturally appropriate Indigenous gatekeeper training program}

As a result of these extensive community consultations, several key components of culturally appropriate gatekeeper training programs were identified (Table 2). Foremost was the need for a long-term sustainable program that enables local Indigenous communities to manage suicide risk. Participants highlighted a need for "culturally acceptable, practical, easily implemented and selfsustainable" gatekeeper training programs. "We need something now, and something that we can easily use and keep our people safe". They suggested the programs use the local Indigenous language and relevant, relatable scenarios to deliver the training in a more effective method. "It has to be for us so we can use it properly and something we can relate to". Supporting technology, such as a mobile app would also help gatekeepers to receive relevant updates. Participants indicated that "we need to learn the technology to make things quicker and use it to help us make safer communities."

Additionally, it was considered that an Indigenousspecific gatekeeper training program should have generalisability to various Indigenous communities in Australia. "Not all communities have the same culture, we have different languages and cultures all across the state and country." A focus on the social, emotional, and spiritual underpinnings of community wellbeing, not just cultural, was also emphasised and deemed necessary for an effective suicide prevention training package. "We need to have the healing and spiritual context included and we want to have them understand what this means for us in our lives." Lastly, it was suggested that the effective components and resources of existing training programs and resources could be integrated into a new culturally appropriate gatekeeper training program. "We really need an Indigenous appropriate training program otherwise there won't be much change."

Table 2 Key components required for a culturally appropriate gatekeeper training package

\footnotetext{
- Short duration

- Practical - easy to use

- Relevant - language \& scenarios

- Sustainable - self-empowering communities

- Generalisable - adaptable across all Indigenous communities

- Inexpensive - available for everyone

- Integration of current valuable resources

- Focus on social, emotional, cultural and spiritual underpinnings of community wellbeing
}

\section{Discussion}

Our study found a lack of community-led suicide intervention training programs that could be effective in Indigenous communities. Previous research has also highlighted the lack of Indigenous-appropriate training programs [21]. Although previous studies have adapted various forms of culturally-modified gatekeeper training programs $[9,10,12]$, they did not use CBPR to develop an Indigenous specific training program that meets community needs. We also found general agreement among participants on the limitations of existing programs and on the need for a culturally appropriate suicide prevention program across all of rural and remote Queensland. A need for systematic evaluation of the cultural appropriateness and effectiveness of Indigenous suicide prevention programs was also highlighted.

Given that suicide occurs after a sequence of events and decisions, prevention should be based on understanding the individual, and the decisions they are making [22]. Intervention strategies with high levels of local Indigenous community involvement are the most effective [13] but also require communities to become self-sufficient and able to both recognize risk factors and implement early intervention. To do this, a culturally-designed, systematic and consolidated Indigenous gatekeeper training program is imperative [21]. Supporting tools using technology [23, 24], may also be beneficial for gatekeepers.

No adolescents $(<18$ year olds) were consulted at this stage of the community consultation, which may be a limitation. Due to the nature of the consultations, talking to adolescents about suicide, suicide prevention and interventions had complex ethical and practical issues and hence, anyone younger than 18 years of age were not included for consultations at this stage.

\section{Conclusion}

Existing models of Indigenous gatekeeper training and other suicide prevention models are not culturally tailored and do not empower Indigenous peoples. Practical suicide prevention programs are needed to mitigate the increasing rates of suicide experienced by Indigenous peoples living in rural and remote locations. Developing such programs requires a community-led approach to identify gaps in models developed for mainstream populations and to identify key elements that would make the program specific to Indigenous communities.

\section{Note}

We respectfully identify Aboriginal and Torres Strait Islander people as Indigenous Australians within this manuscript. 


\section{Acknowledgements}

The National Health and Medical Research Council is acknowledged for their support in funding this project. This project could not have been undertaken without the advice, acceptance, and continued support of the Indigenous communities. Their contribution is acknowledged with gratitude. Acknowledgements are also made to the organisations and community programs listed in Table 1 that provided us valuable time towards this research, and provided consent to be acknowledged for this research.

\section{Funding}

This review was made possible by a Project Grant from the NHMRC (APP1076729) and the Australian Government Department of Health Rural Clinical Training and Support scheme. LH is funded by an Australian Research Council Future Fellowship. SLB-O is funded by a Career Development Fellowship from the NHMRC (APP1107510).

\section{Availability of data and materials}

The data generated and analysed during this study has been stored on restricted databases of The University of Queensland. The data is not available for public sharing due to confidentiality and to protect participant's identity.

\section{Authors' contributions}

BN data collection, analysis, interpretation of findings, manuscript drafting, manuscript editing, approval for submission. SK concept, design, interpretation of findings, critical review of manuscript, approval for submission. LH concept, design, interpretation of findings, critical review of manuscript, approval for submission. GR concept, design, interpretation of findings, critical review of manuscript, approval for submission SBO concept, design, interpretation of findings, critical review of manuscript, approval for submission. GN concept, design, analysis, interpretation of findings, critical review of manuscript, approval for submission. NG concept, design, interpretation of findings, critical review of manuscript, approval for submission. $\mathrm{NH}$ concept, design, interpretation of findings, critical review of manuscript, approval for submission. SKC concept, design, analysis, interpretation of findings, critical review of manuscript, manuscript revision, approval for submission. MT concept, design, analysis, interpretation of findings, critical review of manuscript, approval for submission. All authors read and approved the final manuscript.

\section{Competing interests}

The authors declare that they have no competing interests.

\section{Consent for publication}

Not applicable.

\section{Ethics approval and consent to participate}

The University of Queensland Human Research Ethics Committee approved the study (Approval \#: 2,015,000,662). All participants provided written informed consent to participate in this study.

\section{Publisher's Note}

Springer Nature remains neutral with regard to jurisdictional claims in published maps and institutional affiliations.

\section{Author details \\ ${ }^{1}$ Rural Clinical School, Faculty of Medicine, The University of Queensland, Toowoomba, QLD, Australia. ${ }^{2}$ Departments of Psychiatry, Community Health \& Epidemiology, Dalhouise University, Halifax, Canada. ${ }^{3}$ School of Psychology, Faculty of Health and Behavioural Sciences, The University of Queensland, Brisbane, QLD, Australia. ${ }^{4}$ School of Rural Medicine, University of New England, Armidale, NSW, Australia. ${ }^{5}$ Australian Institute for Musculoskeletal Science (AIMSS), The University of Melbourne, Melbourne, Australia.}

Received: 7 February 2017 Accepted: 6 June 2017 Published online: 13 June 2017

\section{References}

1. ABS. 3309.0 - Suicides, Australia, 2010. In: Canberra: Australian bureau of statistics; 2012.

2. AlHW. Young Australians, their health and wellbeing 2011. In: Canberra: Australian Institute of health and welfare; 2011.
3. AlHW. Suicide and hospitalised self-harm in Australia. In: Canberra: Australian Institute of health and welfare; 2014.

4. AlHW-AIFS. Strategies to minimise the incidence of suicide and suicidal behavior. In: Canberra: closing the gap clearinghouse; 2013.

5. Ridani R, Shand F, Christensen H, McKay K, Tighe J, Burns J, et al. Suicide prevention in Australian Aboriginal communities: a review of past and present programs. Suicide Life Threat Behav. 2015;45(1):111-40.

6. UWA. What we know about suicide prevention for Aboriginal and Torres Strait Islander peoples. In: Aboriginal and Torres Strait Islander suicide prevention evaluation project (ATSISPEP). The University of Western Australia; 2015.

7. Isaac M, Elias B, Katz L, Belik S, Deane F. Gatekeeper training as a preventative intervention for suicide: a systematic review. Can J Psychiatr. 2009;54(4):260-8.

8. Turley B, Tanney B. Living works Australian field trial evaluation report. In: LifeLine Australia \& LivingWorks education inc; 1998.

9. Capp K, Deane F, Lambert G. Suicide prevention in Aboriginal communities: application of community gatekeeper training. Aust N Z J Public Health. 2001:25(4):315-21.

10. Deane F, Capp K, Jones C, Ramirez D, Lambert G, Marlow B, et al. Two-year follow-up of a community gatekeeper suicide prevention program in an Aboriginal community. Aust J Rehabil Couns. 2006;12(1):33-6.

11. LaFramboise $R$, Lewis $H$. The Zuni life skills development program: a school/ community-based suicide prevention intervention. Suicide Life Threat Behav. 2008;38(3):343-53.

12. Sareen J, Isaak C, Bolton S, Enns M, Elias B, Deane F, et al. Gatekeeper training for suicide prevention in first nations community members: a randomized controlled trial. Depress Anxiety. 2013;30(10):1021-9.

13. Clifford A, Doran C, Tsey K. A systematic review of suicide prevention interventions targeting indigenous peoples in Australia, United States, Canada and New Zealand. BMC Public Health. 2013;13:463.

14. Wexler L, Gone J. Culturally responsive suicide prevention in indigenous communities: unexamined assumptions and new possibilities. Am J Public Health. 2012;102(5):800-6.

15. Wexler $L$, White J, Trainor B. Why an alternative to suicide prevention gatekeeper training is needed for rural indigenous communities: presenting an empowering community storytelling approach. Critical Public Health. 2014;25(2):205-17

16. Israel B. Methods in community-based participatory research for health. 1st ed. San Francisco: Jossey-Bass; 2005.

17. Salimi Y, Shahandeh K, Malekafzali H, Loori N, Kheiltash A, Jamshidi E, et al. Is community-based participatory research (CBPR) useful? A systematic review on papers in a decade. Int J Prev Med. 2012;3(6):386-93.

18. Cox A, Dudgeon P, Holland C, Kelly K, Scrine C, Walker R. Using participatory action research to prevent suicide in Aboriginal and Torres Strait Islander communities. Aust J Prim Health. 2014;20(4):345-9.

19. Tong A, Sainsbury P, Craig J. Consolidated criteria for reporting qualitative research (COREQ): a 32-item checklist for interviews and focus groups. Int J Qual Health Care. 2007;19(6):349-57.

20. O'Brien B, Harris I, Beckman T, Reed D, Cook D. Standards for reporting qualitative research: a synthesis of recommendations. Acad Med. 2014;89(9):1245-51.

21. Nasir BF, Hides L, Kisely S, Ranmuthugala G, Nicholson GC, Black E, et al. The need for a culturally-tailored gatekeeper training intervention program in preventing suicide among indigenous peoples: a systematic review. BMC Psychiatry. 2016;16(1):357.

22. Shahtahmasebi S. Indigenous populations and suicide prevention. Int Public Health J. 2014;6(1):33-7.

23. Larsen M, Cummins N, Boonstra T, O'Dea B, Tighe J, Nicholoas J, et al. The use of technology in suicide prevention. In: 2015 37th annual international conference of the IEEE engineering in Medicine and biology society (EMBC): 2015. Milan: IEEE; 2015. p. 7316-9.

24. Luxton D, June J, Kinn J. Technology-based suicide prevention: current applications and future directions. Telemed J E Health. 2011;17(1):50-4.

25. ABS. Australian Statistical geography standard (ASGS) volume 1 - main structure and greater Capital City Statistical areas, July 2016. In: Australian bureau of statistics; 2016. 